\title{
Corrigendum
}

\section{Corrigendum to "Vehicle Detection and Ranging Using Two Different Focal Length Cameras"}

\author{
Jun Liu $(1)$ and Rui Zhang $@$ \\ School of Automotive and Traffic Engineering, Jiangsu University, 212013, China \\ Correspondence should be addressed to Jun Liu; liujun@ujs.edu.cn \\ Received 14 August 2020; Accepted 14 August 2020; Published 7 September 2020 \\ Copyright (c) 2020 Jun Liu and Rui Zhang. This is an open access article distributed under the Creative Commons Attribution \\ License, which permits unrestricted use, distribution, and reproduction in any medium, provided the original work is \\ properly cited.
}

In the article titled "Vehicle Detection and Ranging Using Two Different Focal Length Cameras" [1], there was an error in Figure 2 and Table 1. The authors identified that in the code used for calculating the parameter amount, the number of channels in the last layer of convolution was incorrectly set to an initial value of 255 . The correct value should be 39 , and Figure 2 and Table 1 are corrected to reflect this. The authors apologize for this error and confirm that it does not affect the results and the conclusions of the article. The corrected Figure 2 is as follows:

The corrected Table 1 is as follows: 


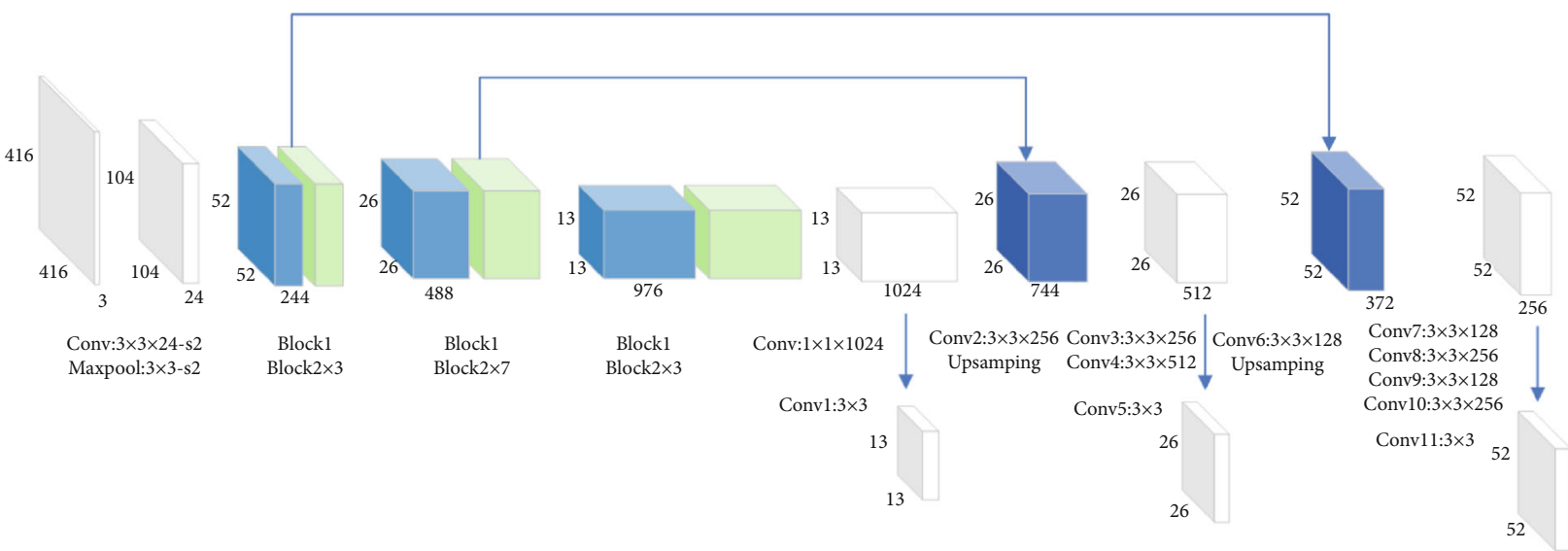

FIGURE 2: Lightweight YOLO network structure.

TABLE 1: Comparison of network detection results.

\begin{tabular}{lccccc}
\hline Network & Classification & Precision $(\%)$ & Recall (\%) & Calculation time per frame (ms) & Parameter number \\
\hline \multirow{2}{*}{ YOLOv3 } & Vehicle & 94.76 & 86.25 & 93.5 & $6.15 \mathrm{e}+7$ \\
& License plate & 91.21 & 82.54 & & \multirow{2}{*}{$1.21 \mathrm{e}+7$} \\
Lightweight YOLO & Vehicle & 90.38 & 82.87 & 74.5 & \\
& License plate & 86.81 & 78.91 & & \\
\hline
\end{tabular}

\section{References}

[1] J. Liu and R. Zhang, "Vehicle Detection and Ranging Using Two Different Focal Length Cameras," Journal of Sensors, vol. 2020, Article ID 4372847, 14 pages, 2020. 